\title{
A new threshold estimation method of SEMG wavelet de-noising for prolonged fatigue identification
}

\begin{abstract}
In sports training, fatigue management is very important to avoid muscle injury and chronic fatigue syndrome.It occurs due to untreated normal fatigue (NF) which leads to a higher level of fatigue. This paper refers to the higher level of fatigue condition as prolonged fatigue (PF). Fatigue can be identified based on musculoskeletal, physiological, psychological, immunological and endocrinal system condition. Presently, musculoskeletal or muscle condition can be accessed quantitatively based on an invasive technique known as biopsies. It is accurate to evaluate muscle condition, but not suitable for frequent measurements. The noninvasive method used through self-evaluation tools such as questionnaire is not a quantitative measurement and sometimes is difficult to quantify. There are other non-invasive methods to evaluate muscle condition known as surface electromyography (SEMG). Before this, SEMG were only widely used to classify between non-fatigue and NF conditions. However, NF prediction might not be accurate and suitable to be used on athletes since they are required to undergo high-intensive training every day and this involves PF condition. Recently, SEMG signals characteristics found out to be able to evaluate ionic concentration changes in the muscle due to intensive training. The signals characteristics are different compared to NF identification. Therefore, high quality SEMG plays an important role in PF detectionto avoid misinterpretation. In this study, twenty healthy participants were recruited and performed five consecutive days of intensive training to induce PF symptoms. The training was based on Bruce Protocol treadmill test and SEMG data were collected fro $\mathrm{m}$ the participants' rectus femoris muscle. This paper pre-processed SEMG signals using Stationary Wavelet Transform (SWT) 'db' 45 with different thresholdestimation techniques of de-noising such as RigRSURE, HeurSURE, minimax, universal threshold and a new estimation of threshold method.The new method able to overcome the limitation of conventional methods which estimate threshold based on statistical principles. Most importantly, the new method can preserve significant SEMG information, remove corner frequency and mitigate baseline noises. The performance of conventional and propose methods can then be evaluated based on PF classification performance. The de-noised signals extracted based on time, frequency and time-frequency features.Naïve Bayes classification results using time and frequency features indicate that the new estimation of threshold method with time and frequency features have the highest accuracy (98\%), compared to RigRSURE (85\%), HuerSURE (68\%), Universal Threshold (74\%) and minimax (76\%) in PF identification.
\end{abstract}

Keyword: De-noising; Wavelet transform; Surface electromyography; Muscle fatigue; Overtraining; Sports 